
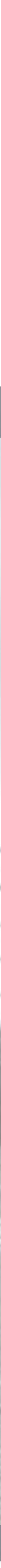

Plaza Roja 

Referencia al citar este artículo:

Mora, P.A., Arias, A.F., Correa, R.A. (2018). Caracterización del perfil en habilidades y capacidades TI en un grupo de muestra de productores de Quinua en el Cauca. REVISTA TEMAS, III(12), 175-186.

\section{Caracterización del perfil en habilidades y capacidades TI en un grupo de muestra de productores de quinua en el Cauca ${ }^{1}$}

Paula Andrea Mora Pedreros ${ }^{2}$ Andrés Fabián Arias Ureña ${ }^{3}$ Rumel Antonio Correa Vargas ${ }^{4}$

\section{Resumen}

Este artículo destaca los avances en el tema de gamificación desarrollado por la empresa Kcumen y los grupos de investigación GICSH y AMAUTAS para la construcción de una plataforma cloud y móvil en un entorno gamificado que consigue un enfoque adecuado del agricultor de la quinua en la implementación en el departamento de Cauca, de tal manera que pueda mejorar su productividad y competitividad en el sector gracias a la industria $\mathrm{Tl}$ y evidenciar la intersección entre educación y tecnología.

\section{Palabras clave}

Plataforma cloud movil, gamificación, productores de quinua, tecnología educativa.

\section{Characterization of the profile in it skills and capabilities in a sample group of producers of quinua in el Cauca}

\section{Abstract}

This article highlights advances in gamification theme developed by the company Kcumen and research groups GICSH and AMAUTAS for the construction of a cloud platform and mobile in an environment gamificated that gets a proper approach of the farmer of the quinoa in the implementation of quinua agricultural practices gap in Cauca, in such a way that can enhance their productivity and competitiveness in the sector thanks to the TI industry.

\section{Keywords}

Cloud plataform mobile, gamification, quinua agricultural, Educative technology.

1. Artículo de investigación.

2. Paula Andrea Mora Pedreros, magíster en Tecnología Educativa. Correo electrónico: paula.mora.p@uniautonoma.edu.co

3. Andrés Fabián Arias Ureña, diseñador gráfico. Correo electrónico: andardg@gmail.com

4. Rumel Antonio Correa Vargas, diseñador gráfico. Correo electrónico: rumelcorrea@unicauca.edu.co 


\section{Introducción}

El proyecto denominado "Plataforma cloud y móvil en un entorno gamificado para la implementación de Buenas Prácticas Agrícolas en productores priorizados de la Cooperativa Departamental de Productores de Quinua del Cauca" es resultado de la investigación del mismo nombre, y tiene como objetivo principal el potencializar la productividad y competitividad del sector agrícola de la quinua y la industria $\mathrm{Tl}$ en el Cauca, mediante la sofisticación y apropiación de una plataforma tecnológica digital escalable, que apoye la implementación de Buenas Prácticas Agrícolas.

Este proyecto pretende a través de la consolidación de una alianza estratégica contribuir al mejoramiento de los procesos agrícolas y aportar al cierre de la brecha digital mediante la sofisticación de una herramienta tecnológica innovadora que adicionalmente aporte a la consolidación y competitividad de la industria TI del Cauca.

El proyecto está concebido a partir de cuatro componentes: Componente 1. Mejoras definidas para la solución tecnológica determinada por el perfil de los productores por intervenir mediante esquemas de innovación abierta entre los actores de la alianza estratégica. Componente 2. Incremento de la competitividad del sector $\mathrm{Tl}$ y el mejoramiento en la implementación de buenas prácticas agrícolas, mediante la sofisticación de una plataforma tecnológica disruptiva. Componente 3. Apropiación de la solución tecnológica que permita su adecuado uso y aprovechamiento en los productores priorizados de quinua, desplegando una estrategia que promueva la demanda de la solución tecnológica en el sector.
Componente 4. Gestión del conocimiento generado durante el desarrollo del proyecto entre la alianza academia, sectores productivos y Estado.

Se ha conformado un equipo interinstitucional que garantice el éxito del proyecto y promueva actividades que permitan de manera eficiente y efectiva generar los beneficios y resultados planteados en este proyecto. Las organizaciones participantes de la alianza son: la empresa de desarrollo tecnológico Kcumen Digital SAS como ejecutora y encargada de la sofisticacion de la plataforma tecnológica; la Cooperativa Departamental de productores de Quinua del Cauca, organización del sector agrícola que agremia a más de 200 productores de quinua en la región, actuando como la entidad beneficiaria y el acompañamiento de aliados estratégicos que aporten desde las diferentes líneas del conocimiento tanto agrícola como tecnológico y científico, sus capacidades para generar investigación aplicada que conlleve la generación de innovación. Estas organizaciones aliadas son: el Centro Regional de Productividad e Innovación del Cauca, Corporación de Incubación y Fomento de Empresas de Base Tecnológica - Cluster CreaTIC y la Corporación Universitaria Autónoma del Cauca.

A continuación, se explicará parte del proceso desarrollado y los avances del proyecto desde los escenarios de diseño del modelo gamificado y el desarrollo tecnológico en la plataforma cloud móvil Certiagro.

\section{Metodología}

El proyecto contempla cuatro fases que se enuncian a continuación, considerando que en la actualidad está en la primera de ellas: 
Fase 1. Articulación de oferta y demanda TIC y Agro

O-1. Definir mediante esquemas de innovación abierta las mejoras de la solución tecnológica, determinado por el perfil de los productores para intervenir.

Algunos de los resultados de este objetivo se pueden enunciar en:

- Un documento de identificación de características priorizadas para el proceso de sofisticación a partir de ejercicios de innovación abierta.

- Un convenio de trabajo colaborativo interinstitucional firmado por los integrantes de la alianza en el foco de especialización TIC y Agro.

- Un artículo científico publicado a partir de la caracterización del perfil en habilidades y capacidades $\mathrm{TI}$ en un grupo de muestra de productores de quinua en el Cauca.

\section{Fase 2. Sofisticación tecnológica}

O-2. Sofisticar la plataforma tecnológica como medio para incrementar la competitividad del sector TI y aportar en la implementación de buenas prácticas agrícolas en la región.

Algunos de los resultados de este objetivo se pueden enunciar en:

- Una plataforma tecnológica cloud y móvil sofisticada para la implementación de buenas prácticas agrícolas.

- Un esquema (proceso) de transferencia tecnológica para la incorporación de tecnologías Open Data y Big Data para solución tecnológica a sofisticar.

- Una plataforma tecnológica para la implementación de buenas prácticas agrícolas incorporando tecnologías de vanguardia en Gamificación, Open Data y Big Data.

- Un documento de términos y políticas de uso de la plataforma tecnológica sofisticada para la implementación de buenas prácticas agrícolas.

\section{Fase 3. Apropiación y Gestión del} conocimiento

O-3. Desplegar una estrategia de apropiación de la solución tecnológica que permita su adecuado uso y aprovechamiento en los productores priorizados de quinua.

Algunos de los resultados de este objetivo se pueden enunciar en:

- Un plan implementado de acompañamiento y formación para la apropiación de la plataforma tecnológica sofisticada para la implementación de buenas prácticas agrícolas.

- Una licencia software de la plataforma cloud y móvil en un entorno gamificado, para la implementación de buenas prácticas agrícolas entregada a la entidad beneficiaria.

- Cinco emprendimientos TI de la región beneficiados mediante la capacitación sobre el desarrollo de soluciones tecnológicas especializadas en el sector agropecuario.

- Una estrategia digital de promoción y difusión implementada, que promueva la demanda y la apertura de nuevos mercados de la solución tecnológica sofisticada en el sector de especialización.

\section{Fase 4. Documentación y registro}

O-4. Gestionar el conocimiento generado mediante su documentación, durante el desarrollo del proyecto alianza entre 
la academia, los sectores productivos y el Estado.

Algunos de los resultados de este objetivo se pueden enunciar en:

- Un proceso de registro de propiedad intelectual y derechos de autor de la solución tecnológica sofisticada en el sector de especialización.

- Una plataforma web para la gestión del conocimiento que permita almacenar y disponer el acceso abierto a la información generada del proyecto.

- Una plataforma web alimentada a partir de los resultados generados de la investigación durante la ejecución del proyecto.

Considerando todas estas variables se diseñó un modelo canvas (Trejos, 2016) para la configuración del proyecto.

Figura 1. Modelo Canvas de desarrollo del proyecto

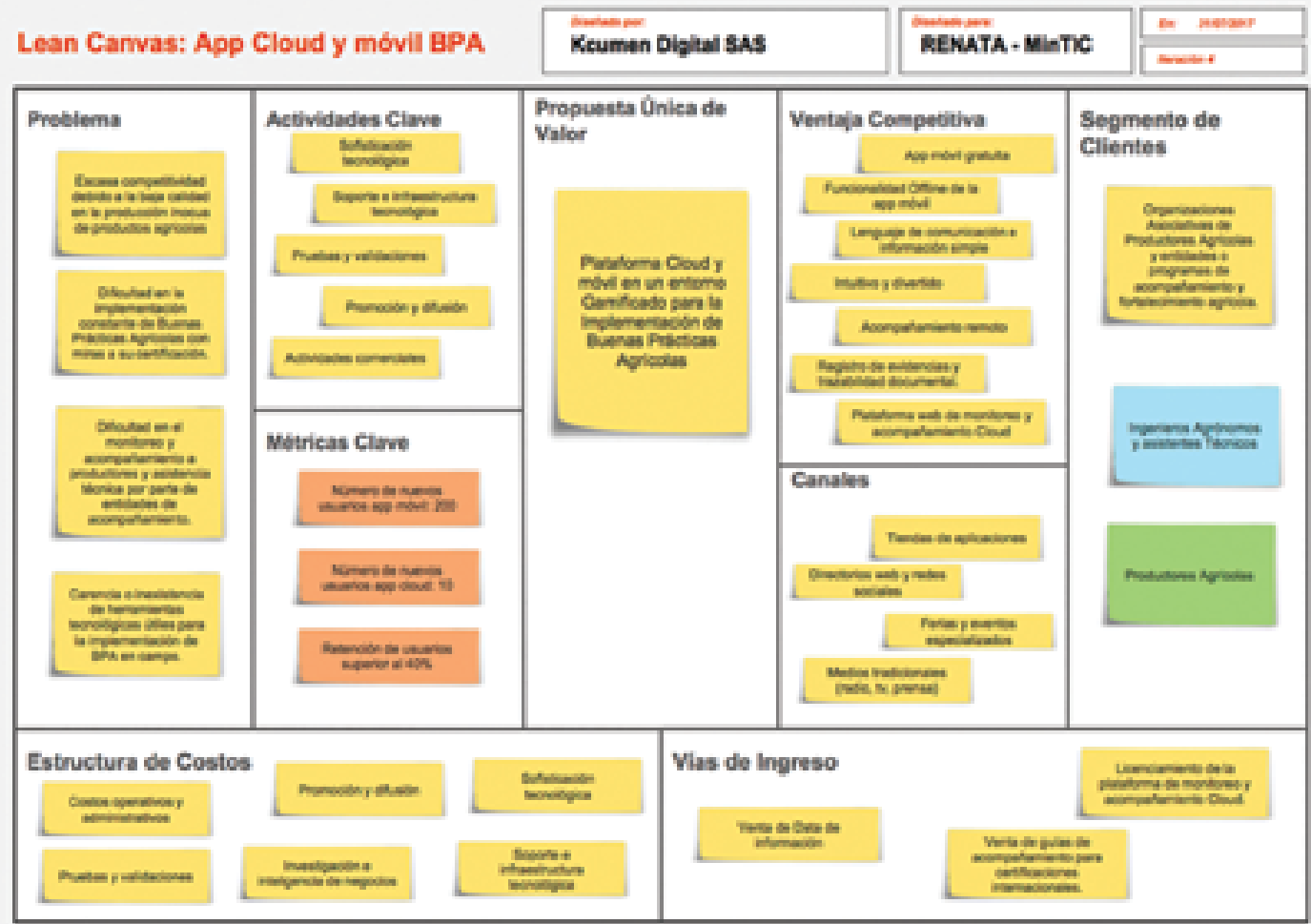

Fuente: Trejos, M., 2016. Modelo canvas de desarrollo del proyecto.

\section{Plataforma de implementación Buenas Prácticas Agrícolas (BPA)}

La Plataforma para la implementación de BPA es un sistema tecnológico basado en tecnologías móviles y cloud, que cuenta con un alto componente de innovación por el uso de sistemas de gamificación, interfaces intuitivas, uso de tecnologías de desarrollo de software de vanguardia, que pretende convertirse en una útil herramienta para aportar a los productores agrícolas para que mejoren sus procesos de producción a través de una serie de pasos que aportan a la inocuidad de los productos agrícolas cultivados (Díaz, 2008). 
Esta solución tecnológica para la implementación de buenas prácticas agrícolas hace parte de una siguiente fase del producto CafetHéroe (Cluster CreaTIC, 2017) que nació como respuesta a la necesidad de los productores de cafés especiales en el departamento del Cauca para incrementar la calidad en la producción de su café. Esta herramienta fue ganadora en el concurso departamental Hackaton Agro 2015, en este evento participaron empresas desarrolladoras de software y expertos temáticos en diferentes líneas del agro regional.

Para el desarrollo de la actual herramienta se considera un aplicativo móvil para dispositivos Android, celulares o tabletas de gama media y alta, funcional en aproximadamente el $95 \%$ de equipos actuales en el mercado, cuenta con una funcionalidad casi total sin necesidad a conexión de internet. Esta herramienta, una vez instalada, le permite al productor agrícola contar con una guía simple e intuitiva para la implementación en su predio, considerando los criterios necesarios para aplicar a la certificación en BPA del ICA.

La aplicación permite que el productor registre la evidencia en el cumplimiento de cada criterio, mediante el uso de fotografías tomadas con su mismo dispositivo, estas evidencias dan cumplimiento de los criterios de la certificación, y en la aplicación se puede evidenciar el progreso en la implementación general del sistema, así mismo el cumplimiento de varios logros; bonifica al productor dándole reconocimientos e insignias que estimulan el uso de la aplicación y por ende estar más cerca de obtener la Certificación.

De igual forma, esta plataforma cuenta con un prototipo web que permite gestionar diferentes tipos de certificaciones agrícolas y asignarlas a un productor en específico o a un grupo de ellos.

Cuenta con una conexión BPA BOT, es un prototipo desarrollado y conectado al sistema automatizado que posibilita, mediante el uso de aplicaciones de mensajería tipo WhatsApp, asignar tareas en una fecha establecida para un productor por parte de su asistente técnico, para este sistema hacemos uso de una plataforma de mensajería llamada Telegram.

Al considerar los aspectos de inversión es importante mencionar que esta plataforma ha tenido una inversión aproximada de $\$ 75.000 .000$ de pesos a partir de la inyección de recursos propios de la empresa y privados, también mediante la financiación de convocatorias de proyectos de regalías regionales, recursos de premios de concursos como Hackaton Agro Cauca, aporte en especie en procesos de acompañamiento tecnológico por parte de CreaTIC.

Actualmente, el prototipo móvil funcional de la plataforma tecnológica para la implementación de buenas prácticas agrícolas, se ha comenzado a validar con aproximadamente 20 usuarios productores del sector de la quinua, en este proceso se han podido realizar 3 iteraciones mejorando aspectos técnicos, de diseño y de concepto.

Así mismo, hemos identificado que la herramienta ha generado una significativa aceptación y a pesar de que no es una herramienta para todos los productores, la mayoría de quienes cuentan con un dispositivo móvil han usado la herramienta de manera bastante positiva, viéndose sobre todo un uso elevado en jóvenes $y$ adultos de entre los 14 y 35 años.

Las entrevistas y pruebas han evidenciado que el mayor esfuerzo durante el proceso de implementación de los crite- 
rios de certificación en BPA (Aguilar, 2009) es el diligenciamiento de la información, actualmente la plataforma no ofrece esta funcionalidad, y es precisamente la intención de esta propuesta: el mejoramiento de esta funcionalidad en el prototipo.

Muchas organizaciones han solicitado el poder tener una plataforma cloud (Jaramillo, Rodríguez, Guzmán, 2007) que permita realizar el monitoreo de los procesos implementados por los productores incluyendo la asistencia técnica. La solución tecnológica aún se encuentra en proceso de desarrollo y esperamos poder contar con una versión 1.0 para iniciar con el proceso de registro de propiedad intelectual, cabe aclarar que los derechos de explotación pertenecen en su totalidad a la empresa Kcumen Digital SAS.

Concordante con lo anterior la plataforma de BPA cuenta con varios atributos diferenciadores y en algunos casos únicos como producto digital para el acompañamiento en la implementación de Buenas Prácticas Agrícolas a productores. Dichos atributos han considerado procesos formativos intuitivos e innovadores como la gamificación, la contextualización del usuario, aquí algunos de ellos:

- Aplicación móvil que no depende de conexión a internet para su funcionamiento (Hassan, Fernández, 2003).

- Entorno intuitivo mediante una interfaz gráfica de uso sencilla. Que facilita la motivación y la interacción con el usuario en procura de un aprendizaje significativo (Rodríguez, 2016).

- Entorno gamificado que estimula y mantiene la retención de los usuarios y la consecución de logros que fomentan su uso constante (Marczewski, 2013).
- Lenguaje e información clara y sencilla orientada al tipo de perfil del público al que va dirigido (Katz, 1998).

- Uso de BOT para la consulta y asignación de tareas a un productor por parte de la asistencia técnica haciendo uso de aplicaciones de chat como Telegram.

- Registro de evidencias de cumplimiento de los criterios implementados. Desarrollo de la habilidad de sistematización (Rodríguez, 2016).

- Almacenamiento Cloud de las evidencias y avances una vez se cuente con conexión a internet (Sander \& Simons, 2009).

Esta solución $\mathrm{Tl}$ como modelo de negocio busca convertirse en una herramienta para el acompañamiento en la implementación de varios tipos de certificación agrícola como, Rainforest, GlobalGAP, ISO, Fairtrade, ICA, entre otras (Herrera, 2015).

\section{Sensibilización y caracterización}

Considerar los escenarios de sensibilización es una de las primeras acciones en la Fase 1, Para este propósito, fue necesario consultar en algunas organizaciones agrícolas que operan en la ciudad de Popayán como Ashofrucol, Crepic, ICA e instancias gubernamentales como la Secretaría de Agricultura del Cauca, con el fin de detectar las zonas productivas en el departamento y establecer comunicación con algunos representantes del gremio.

Los resultados de este acercamiento evidenciaron algunas zonas con mayor actividad productiva; así mismo, existe un ente encargado de agremiar a los productores de quinua, promoviendo la rentabilidad y el crecimiento del producto en el departamento, conocida como la 
Cooperativa Departamental de Productores de Quinua en el Cauca (Jacobsen \& Sørensen, 2010). En este punto, la indagación ayudó a comprender problemas y antecedentes que han afectado negativamente la actividad productiva de los campesinos, como el difícil de acceso a los predios, la poca cobertura de internet, y la disminución del cultivo de quinua por el bajo precio en el mercado.

Algunos de estos factores se deben a la polarización del mismo gremio dentro de la cooperativa, principalmente por la deserción y migración a otros productos en los predios o las pocas garantías de venta y rentabilidad para los campesinos. Estos problemas se agudizan con el paso del tiempo y se ve reflejado en la disminución de asociados en la cooperativa, razones por las cuales es necesario desarrollar acciones y proyectos que reactiven las actividades de producción y comercialización. En este sentido, el proceso de certificación en Buenas Prácticas Agrícolas se convierte en un proceso sumamente importante e indispensable para ampliar las oportunidades comerciales e independientes en los gremios de producción.

En este punto, se hizo el desplazamiento hacia el municipio Los Milagros Cauca, lugar que congrega la mayor parte de afiliados a la Cooperativa. En este primer encuentro se logró trabajar con el apoyo de una representante de la Cooperativa para reunir a los productores afiliados y contextualizar el proyecto; así, se da inicio al trabajo de campo con la muestra seleccionada para conocer las dinámicas productivas de la zona.

El ejercicio de caracterización consideró los siguientes datos significativos: el inicial de ellos fue el género, pues el $70 \%$ son hombres y el $30 \%$ mujeres, con un nivel educativo básico entre primaria $32 \%$ y secundaria $19 \%$, con tan solo un $3 \%$ en educación superior, esa información está visible en la tabla 1. Al ahondar en el ejercicio de uso de las BPA, se encontró la siguiente información que da cuenta de su conocimiento y certificación, derivando también su motivación.

Tabla 1. Identificación BPA

\begin{tabular}{cccccc}
\multicolumn{3}{c}{ Identificación BPA } \\
$\begin{array}{c}\text { Conocimiento } \\
\text { BPA(\%) }\end{array}$ & $\begin{array}{c}\text { Certificación } \\
\text { BPA }(\%)\end{array}$ & $\begin{array}{c}\text { Interés en } \\
\text { certificación } \\
\text { BPA (\%) }\end{array}$ \\
Sí & No & Sí & No & Sí & No \\
30 & 70 & 0 & 100 & 100 & 0
\end{tabular}

Fuente: Esta investigación.

Como se puede apreciar hay un conocimiento sobre la norma, pero no una ejecución real considerando la ausencia total de certificación.

Consecuente con la información anterior se procedió a medir la capacidad tecnológica instalada del agricultor para estimar el impacto de uso de la tecnología en el desarrollo del proceso de formación de sus habilidades, como lo especifica la Figura 2, inicialmente pudo establecerse el $78 \%$ de ellos tienen celular, pero el $65 \%$ carece de un smartphone, limitando el acceso a un rango significativo de aplicaciones para trabajar, igual comportamiento evidenció la existencia de internet en el predio solo el $3 \%$ tiene acceso.

Todo lo anterior nos entrega datos importantes que son considerados en la ruta del modelo de gamificación, a fin de establecer estrategias digitales $y$ complementarias desde lo análogo y la optimización en el uso del celular, es por ello que indagamos igualmente sobre la usabilidad del recurso, y el mayor rango está en llamar o recibir llamadas 54\%. 
Figura 2. Usos alternativos del celular por parte de los productores.

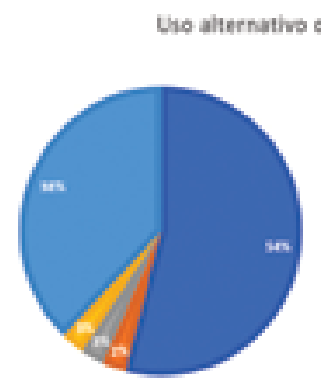

Fuente: esta investigación

Considerando los datos recolectados se procedió a establecer y delimitar el grupo que será atendido en una Fase 2 de caracterización, con quienes podremos pasar a establecer el esquema de gamificación contemplando las mayores ventajas frente al acceso tecnológico y a sus habilidades digitales.

\section{Tecnología y educación desde una Innovación Abierta}

Para fortalecer los procesos de sofisticación (Sanders \& Simons, 2009) y teniendo en cuenta la dimensión del proyecto, es necesario un proceso de ideación interdisciplinario que contenga aportes desde el diseño, la innovación, la investigación, relación con el contexto y desarrollo tecnológico. Es por ello que hablar de innovación abierta nos obliga a considerar un nuevo esquema de pensamiento, en el cual los actores involucrados son generadores de contenidos e ideas potenciales desde su campo de acción, como se grafica en la figura 3. Por lo tanto, combinar los conocimientos internos (empresa) con los externos (disciplinas, grupos de investigación, experiencias técnicas y conocimientos del sector agro) constituyen la base fundamental para enriquecer la experiencia a partir del conocimiento colectivo y la cocreación en todas las fases del proyecto.
Figura 3. Actores del proyecto bajo el esquema de innovación abierta

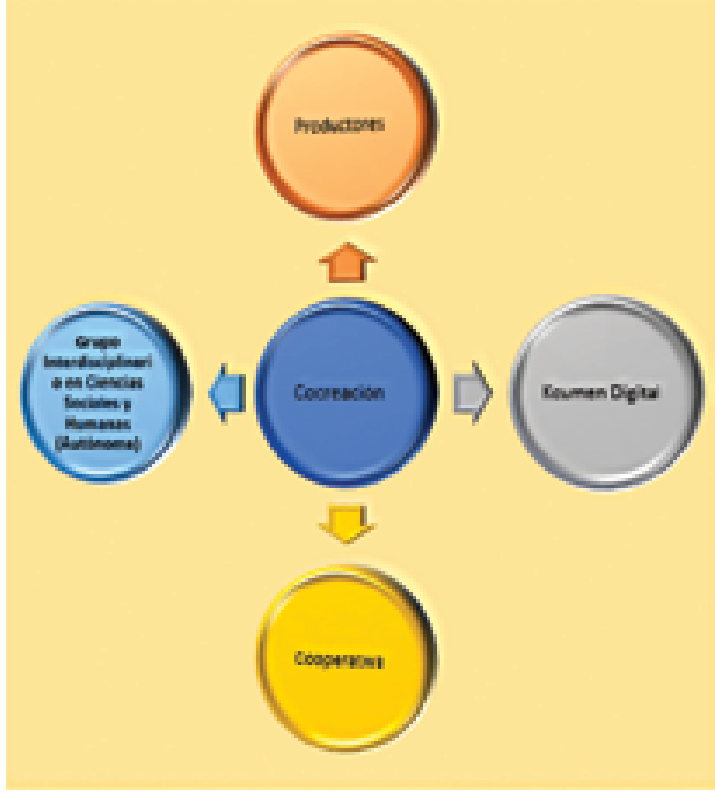

Fuente: Esta investigación

\section{Cocreación}

Uno de los factores fundamentales de la innovación abierta es la participación de todos los actores del proyecto. Esta inclusión contempla la mirada de cada persona dentro de sus experiencias y saberes, proporcionando un ejercicio holístico e interdisciplinar, fundamental en el quehacer de la innovación; dichos actores aportan significativamente a la viabilidad del proyecto. Estas actividades no solo nos ayudan a evaluar las estrategias y diseños desde una mirada inicial; sino desde la perspectiva de los productores y organizaciones, quienes opinaban o sugerían en el mismo instante en que socializamos las propuestas. Esto nos llevó a pensar que la acción de cocrear implicaba considerar cada opinión o sugerencia de los actores del proyecto, si entendemos la co-creación como "cualquier acto de creación colectiva que se experimenta en forma conjunta por dos 
o más personas" (Rodriguez, 2016, p. 78). El modelo propuesto por los autores considera algunos requisitos previos que dan paso a la práctica de la cocreación con un sentido de transformación social de acuerdo con lo expuesto por varios autores incluidos Cruz y Pérez (2009) son:

- La creencia de que todas las personas son creativas y participarán en un proceso si están motivados y se proporcionan las herramientas para hacerlo.

- La diversidad es un factor clave: si todos los participantes son del mismo fondo, la perspectiva y opinión del resultado puede ser limitado e incluso predecible.

- Definición y resolución de problemas en conjunto, son los conductores en el inicio difuso del proceso de diseño.

- El diálogo continuo y la conversación, junto con talleres, son necesarios para ampliar la participación.

- Es necesaria la exploración y utilización del diseño de herramientas, materiales y métodos que ponen a todos los jugadores en un terreno común.

De acuerdo con Sanders (2009), nuestro propósito es mantener la comunicación e interacción con todas las personas involucradas en esta fase de trabajo para construir una herramienta pensada para los usuarios y con los usuarios.

\section{Esquema metodológico de gamificación}

Para el acercamiento metodológico es indispensable la construcción de una estructura general que contemple: conceptos, autores de gran trayectoria en el tema de gamificación, modelos medidos y aplicaciones prácticas para mejorar la experiencia de uso (Staubitz et al., 2014). A modo general, la plataforma busca que los agricultores hagan la implementación de las BPA según la Norma 30021 del ICA, llevando a cabo la interpretación de los contenidos técnicos actuales de dicha norma a un lenguaje cercano y fácil de comprender para el campesino. Este punto se complementa con otro aspecto fundamental, que es la accesibilidad de los contenidos (Austín, 1993), a través de su viabilidad técnica y de navegación, trabajada de manera transversal en todo el proceso de usabilidad. Por otro lado, se procura fomentar el papel de agro empresario (Cruz, Perez, 2009). un concepto que despierta la importancia de incluir a los productores en los procesos productivos desde el campo a la industria, generando contenidos de interés sobre las ventajas de la comercialización y exportación para su benéfico directo. Paralelo a estos retos, es necesario mantener la motivación intrínseca y extrínseca (Bartle, 1996), que permita evidenciar beneficios en corto y largo plazo, una vez comiencen su interacción con el sistema. Es por ello que el análisis abordado desde los retos, resultados e indicadores evidencian el esquema general que da paso al target o usuario, figura 4 que identifica el esquema general de retos, resultados e indicadores, para segmentar los perfiles de usuarios en el esquema gamificado. 
ESQUEMA

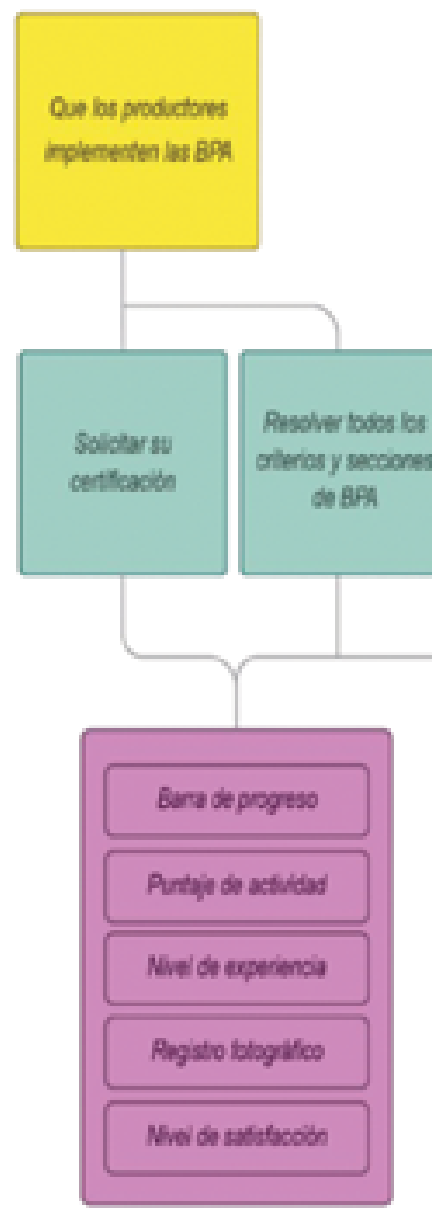

$\square$ RETOS
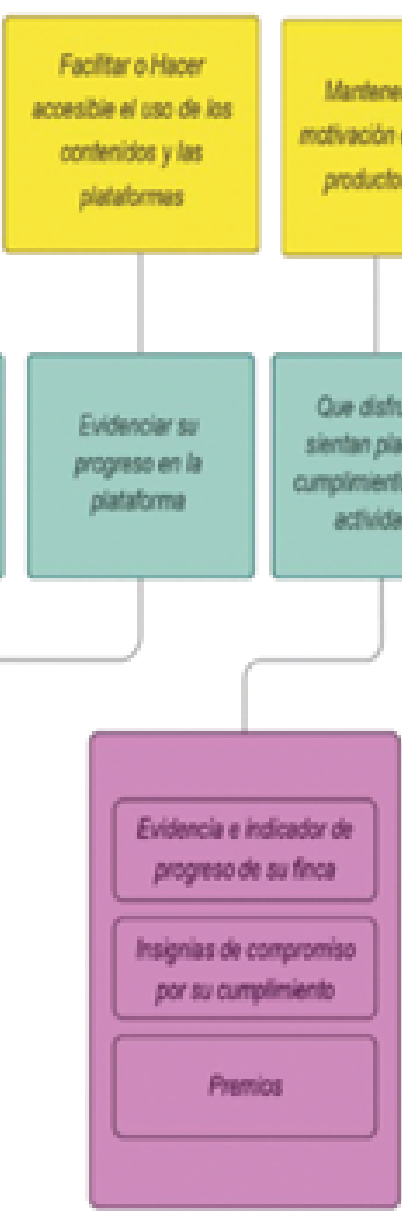

RESULTADOS
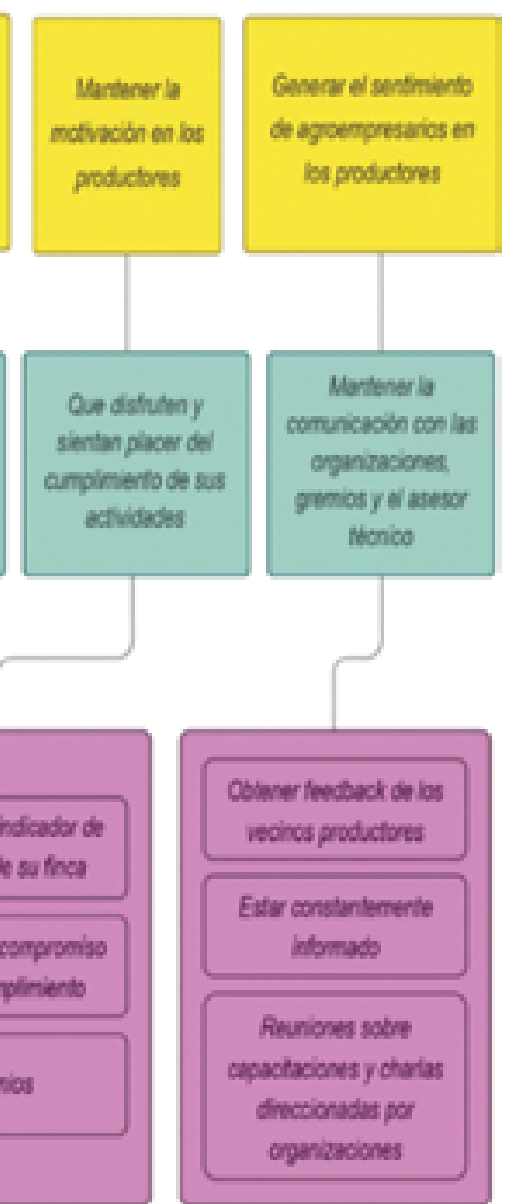

Fuente: Esta investigación.

Cabe aclarar que en todo este ejercicio se procedió a utilizar la teoría de segmentación de usuario RAMP (Relatedness, Autonomy, Mastery, Purpose) (Bartle, 1993).

Para finalizar la presentación de los avances alcanzados, es ineludible considerar lo mencionado anteriormente sobre la segmentación de usuario, dos autores fueron relevantes en la construcción del modelo final; inicialmente, Ricard Bartle (1996) desde los perfiles de jugadores y sus comportamientos en los videojuegos tipo MUD (multi-user dungeons), obedeciendo a dos variables que presentan los sistemas de juego:

- Jugadores vs mundo

- Interacción vs acción

Sobre estos dos ejes, Bartle clasifica cuatro perfiles de jugador: Killer (competitivos) Socialites (sociales) Achievers (triunfadores) Explorers (exploradores). 
Por otro lado, uno de los primeros autores que señala la distinción de jugadores, Andrzej Marczewski (2013) en su artículo "¿Cuál es la diferencia entre la gamificación y los juegos serios?". Marczewski (2013) plantea cuatro tipos de sistemas para diferenciar la gamificación de otras experiencias de juego. Con esta premisa, la teoría de segmentación de Marczewski (2013) retoma los ejes de Bartle (1996) (Jugadores vs mundo -Inte- racción vs acción) para construir perfiles de usuarios acordes al sistema de gamificación, el cual los denomina metodología RAMP (Relatedness, Autonomy, Mastery, Purpose).

Con ello se estableció la metodología RAMP (Bartle, 1996) un esquema de caracterización de usuario para el proceso de gamificación para implementar como se grafica a continuación en la figura 5 .

Figura 5. Esquema de caracterización de la metodología RAMP
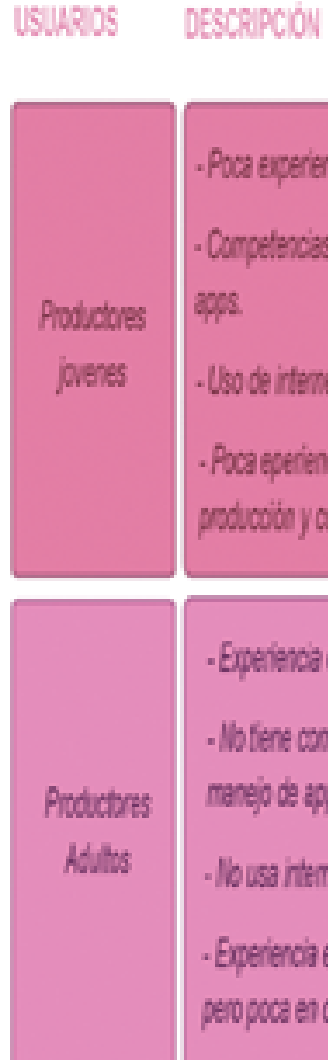

Fuente: Esta investigación.

\section{Conclusiones}

1. Considerar el contexto de acción de los productores de quinua es indispensable para la implementación de una innovación tecnológica y de ahí
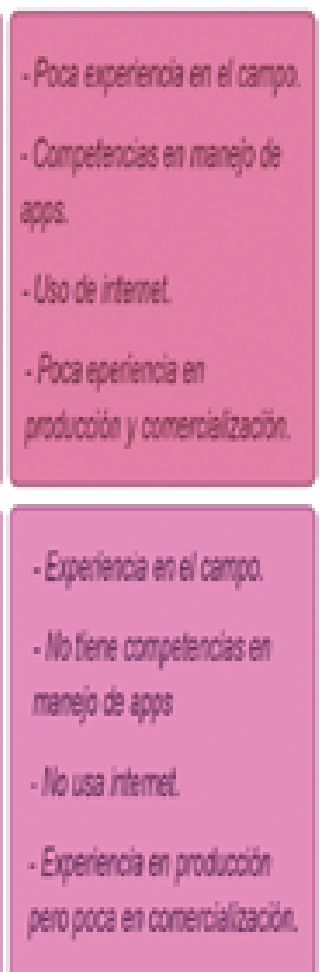
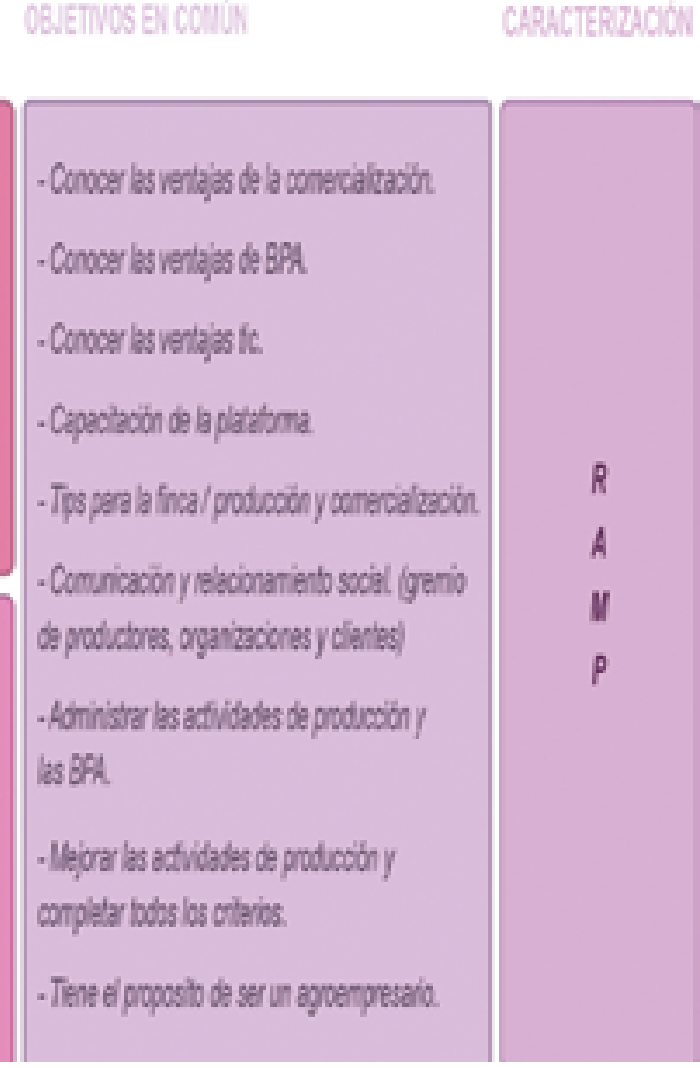

que las fases de caracterización son indispensables.

2. Los perfiles de usuario identificados determinan la brecha generacional evidente en otras investigaciones y su impacto en el uso tecnológico y la 
innovación, es ventaja del proyecto el considerar no solo a productores adultos propietarios de predio sino también a su núcleo familiar en el cual aparecen productores jóvenes con mayor interés sobre el manejo de aplicaciones.

3. La gamificación permite el mejoramiento en la interacción usuario y aplicación de forma que combinamos sus intereses particulares y motivación intrínseca con sus habilidades e intereses por la certificación BPA en procura de una mejor proyección de su quehacer agricultor.

\section{Referencias}

Aguilar, L. J. (2009). La computación en nube (Cloud Computing): El nuevo paradigma tecnológico para empresas y organizaciones en la Sociedad del Conocimiento. Icade. Revista de las Facultades de Derecho y Ciencias Económicas y Empresariales, (76), 95-111.

Austin, J. (1993). El agroempresario del futuro. Revista del Plan Agropecuario 21.

Bartle, R. (1996). Hearts, clubs, diamonds, spades: Players who suit MUDs. Journal of MUD research, 1(1), 19.

Díaz, A. (2008). Buenas Prácticas Agrícolas: guía para pequeños y medianos agroempresarios. Serie de Agronegocios. Cuadernos de Exportación, (11 Tegucigalpa).

Hassan, Y., \& Martín, F.J. (2003). Qué es la accesibilidad web. No solo usabilidad, (2).
Herrera, D.C.F. (2015). El modelo Canvas en la formulación de proyectos. Cooperativismo \& Desarrollo, 23(107), 118-142.

Izquierdo, J., Rodriguez Fazzone, M., \& Durán, M. (2007). Manual de buenas prácticas agrícolas para la agricultura familiar.

Jacobsen, S.E., \& Sørensen, M. (2010). Quinua y su producción en Bolivia: de éxito económico a desastre ambiental. Esta publicación cuenta con el apoyo financiero de, 85.

Jaramillo, J., Rodríguez, V.P., Guzmán, A., \& Zapata, M.A. (2007). Manual técnico Buenas Prácticas Agrícolas (BPA) en la producción de tomate bajo condiciones protegidas. Corpoica (No. Doc. 22213). CO-BAC, Bogotá).

Katz, J. (1998). Aprendizaje tecnológico ayer y hoy. Revista de la CEPAL.

Marczewski, A. (2013). What's the difference between gamification and serious games. Andrzej's Blog.

Martín, N., Martín, V., \& Trevilla, C. (2009). Influencia de la motivación intrínseca y extrínseca sobre la transmisión de conocimiento. El caso de una organización sin fines de lucro. CIRIEC-España. Revista de Economía Pública, Social y Cooperativa, (66).

Rodriguez, D. (2016). Entornos post-digitales, cocreación, prácticas didácticas y experiencias de usuario. En VIII Jornadas de Investigación en Disciplinas Artísticas y Proyectuales (La Plata, 6 y 7 de octubre de 2016).

Sanders, L., \& Simons, G. (2009). A social vision for value co-creation in design. Open Source Business Resource.

Staubitz, T., Woinar, S., Renz, J., \& Meinel, C. (2014, november). Towards social gamificationimplementing a social graph in an xmooc platform. In Proceedings of the 7th International Conference of Education, Research and Innovation. Seville, (pp. 17-19). Spain.

Trejos, M. (2016). Planteamiento y evaluación de sistemas productivos agrícolas en áreas inundables del río Cauca. (Doctoral dissertation). 